\title{
7 Differenzierungen zwischen geschlossener Umwelt und offener Welt: Zum Verhältnis zwischen der Philosophischen Anthropologie und Uexkülls neuer Biologie der Umwelten
}

\subsection{Drei systematische Schwerpunkte von Plessners Auseinandersetzung mit Uexküll}

Helmuth Plessner verweist schon im Vorwort zur ersten Auflage seiner Naturphilosophie Die Stufen des Organischen und der Mensch. Einleitung in die philosophische Anthropologie (1928) auf die „neue Biologie“, als deren entscheidenden Vertreter (neben Hans Driesch) er Jakob Johann von Uexküll (Plessner 1975, III) nennt. Unter der neuen Biologie wird, wie sich im ganzen Buch zeigt, eine moderne, weil experimentell verfahrende Erfahrungswissenschaft verstanden, die aber im Unterschied zu Physik und Chemie der Spezifik des Lebendigen gerecht zu werden versucht. Dabei wird von Plessner für die Biologie die an Darwin anschließende Evolutionstheorie grundsätzlich anerkannt, aber ihre darwinistische Einseitigkeit kritisiert. Diese Einseitigkeit bestehe darin, das Verhältnis zwischen Organismus und Umwelt primär als einen Kampf ums Dasein aufzufassen, in dem das jeweilige Lebewesen primär in einer gegenüber seiner Umwelt passiven Rolle erscheine, da es sich ihr anzupassen habe und von ihr selegiert werde. Aber selbst wenn man sich allein an die Evolutionstheorie hielte, müsste man in ihr mit gleichem Recht neben dem Kampf ums Dasein auch die Eintracht, die Konkordanz oder die Eingespieltheit zwischen Lebewesen und Umwelt berücksichtigen. Zudem gelte es, sowohl die Anpassung als auch die Selektion nicht nur von der Umwelt her zu begreifen, sondern ebenso aktiv vom Organismus ausgehend. Uexkülls neue Biologie habe ihre Stärke darin, das Gegengewicht zu den darwinistischen Verkürzungen darzustellen, nämlich den „Bauplan“ des jeweiligen Organismus freizulegen und so seine ihm spezifische Umwelt erschließen zu können, in der er aktiv zu werden vermag. Allerdings werde Uexkülls Gegenposition ebenso einseitig wie der darwinistische Fokus, nur in der umgekehrten Richtung.

Zweitens bleibe Uexkülls grundsätzliche Kritik an der anthropomorphen Übertragung des Selbstverständnisses von Menschen auf Tiere berechtigt, weil diese Kritik für die Biologie als überprüfbare Experimentalwissenschaft eintrete, so Plessner schon 1925 (Plessner 1982c, 80f.). Uexkülls Kritik betraf vor allem die „Anthropozentrik“ und erfolgte dagegen positiv im Namen einer „maschi- 
nellen Biologie“, die sich mit Strukturen und funktionalen Leistungen der Lebewesen beschäftigt (Uexküll 2014, 42, 62f.). Uexküll hatte das Reiz-ReaktionsSchema über den Behaviorismus hinaus auf Verhaltensfunktionen hin geöffnet, die er im jeweiligen Bauplan für die Einheit des Organismus mit seiner Umwelt darstellen konnte. Zugleich hatte Uexküll um den übermaschinellen Charakter lebendiger Ganzheiten gewusst, wenngleich diesen Charakter unter Verweis auf das Protoplasma einer „technischen Biologie“ (ebd., 63) überlassen. Was Plessner indessen - bei aller Gemeinsamkeit im Problembewusstsein - an Uexküll kritisierte, bestand nun darin, dass Uexküll noch nicht in die leibliche Zwischenschicht des Verhaltens zwischen dem Organismus und seiner Umwelt vorgedrungen sei. Die Thematisierung der leiblichen Zwischenschicht, die psycho-physisch neutral sei, war für Plessner aber möglich und nötig, vor allem, um zwischen den Verhaltensleistungen niedriger und höher entwickelter Tiere, darunter insbesondere der Intelligenz der Anthropoiden, differenzieren zu können. Daher interpretierte Plessner Uexkülls Programm der Erforschung von Bauplänen so um, dass es sich hierbei nur um ein Minimalprogramm, nicht aber um ein Maximalprogramm handeln kann. Dadurch kann Plessner im Gegensatz zu Uexküll für die höher entwickelten Tiere an die Tierpsychologie von Wolfgang Köhler und Frederick Jacobus Johannes Buytendijk anschließen. Uexküll hielt diese Tierpsychologie für Psychologie, nicht aber für Biologie (Uexküll 1973, 215).

Schließlich versagte aus Plessners Sicht Uexkülls mangelhafte Unterscheidung zwischen Welt und Umwelt, die eher auf eine Parallelisierung von verschiedenen Umwelten hinauslaufe. Uexkülls Nivellierung betreffe den philosophisch-anthropologischen Unterschied zwischen der intelligenten Lebensweise von Menschenaffen, deren Intelligenz Wolfgang Köhler zweifellos nachgewiesen habe, und dem mitweltlichen Geist in der personalen Lebenssphäre von Menschen. Plessner kritisierte aber zugleich den Fehler, der sich aus der Umkehrung von Uexkülls biologischer Pluralisierung aller für in der Natur als gleichranging gehaltenen Umwelten ergibt: Die kategoriale Unterscheidung zwischen Umwelt und Welt hatte zu dem ebenso falschen Gegensatz geführt, als seien nur Tiere an eine bestimmte Umwelt gebunden, während das personale Leben in der Offenheit von Welt stehe. Plessner bestand darauf, dass das personale Leben von Menschen sowohl einer biotischen als auch einer soziokulturellen Umwelt bedürfe. Die personale Lebenssphäre werde systematisch gesehen aus einer Eröffnung von Welt heraus ermöglicht, wodurch sie nur geschichtlich geschaffen, eingerichtet und verändert werden könne. Daher dürfe man die Umweltgebundenheit von Tieren nicht mit denjenigen soziokulturellen Umwelten kategorial gleichsetzen, die in der personalen Lebenssphäre aus Weltoffen- 
heit heraus eingerichtet werden. Nach der Grundlagendiskussion der 1920er Jahre tauchte dieses Problem falscher Gleichsetzungen dramatisch in der rassistischen Ideologie des Nationalsozialismus auf (Plessner 2001d), dann aber erneut auch in der gesamten Nachkriegszeit, in der dieser Kategorienfehler ideologiebildend fortwirkte (Plessner 1983j u. 2001b). Die Verschränkung von Umwelt und Welt im personalen Leben im Unterschied zur Umweltabhängigkeit von Tieren stand im Mittelpunkt des von Plessner organisierten interdisziplinären Philosophie-Kongresses 1950, an dem auch Biologen und Mediziner teilnahmen (Plessner 1983k), und der großen interdisziplinären Propyläen Weltgeschichte (1961), die von Golo Mann und Alfred Heuß herausgegeben wurde, die Plessner gebeten hatten, die philosophisch-anthropologische Einleitung in diese Weltgeschichte zu schreiben (Plessner 1983e).

Damit ergeben sich im Überblick drei Schwerpunkte in Plessners Auseinandersetzung mit Uexkülls Biologie, nämlich 1. die Überwindung darwinistischer Einseitigkeiten in der Ausarbeitung eines weiten Spektrums evolutionstheoretischer Erklärungsmöglichkeiten, ohne sich selber von der umgekehrten Vereinseitigung durch Uexküll abhängig zu machen, 2. Uexkülls berechtigte Kritik am Anthropomorphismus nicht mit einem Maximalanspruch seines eigenen Biologieverständnisses zu verwechseln, sondern sein Forschungsprogramm im Vergleich mit der Tierpsychologie auf einen Minimalanspruch zu begrenzen, und 3. den Tier-Mensch-Vergleich so umzustellen, dass biotische nicht mit soziokulturellen Umwelten gleichgesetzt werden, wie es in biologistischen Ideologien geschieht, während die Biologie aber als Wissenschaft einen philosophischen Weltbegriff voraussetze. Alle drei Schwerpunkte durchziehen alle Schriften Plessners, in denen er zwischen 1925 (Plessner 1982c) und 1965 (dem Vorwort zur zweiten Auflage von Plessner 1975) auf von Uexküll verweist, in verschiedener Kombination. Diese systematischen Schwerpunkte werden im Folgenden in der problemgeschichtlichen Reihenfolge der wichtigsten Schriften von Plessner zu Uexküll erörtert.

Die durchlaufende Systematik der Schwerpunkte ergibt sich aus Plessners durchgängig philosophischem Anliegen. Als Mitarbeiter von Buytendijk war Plessner zwar auch als empirischer und theoretischer Biologe tätig, so dass er diese Diskussionen aus teilnehmender Beobachtung verstehen konnte, aber er hat nicht darin sein originäres Anliegen gesehen. Plessner verhält sich zu Uexkülls Biologie nicht als Biologe, sondern als Philosoph der lebendigen Natur (Plessner 1975, 24, 26, 77). Er begründet eine „philosophische Biologie“ (ebd., III, 66, 76), auf deren Umweg eine „philosophische Anthropologie“ (ebd., IV, 30-36) gewonnen werde, um den anthropologischen Zirkel zu vermeiden. Diese Philosophie ziele auf die kategoriale Rekonstruktion qualitativer Erfahrungen 
(d. h. Anschauungen) in der personalen Lebensführung ab, die Laien und Experten miteinander in einem aufgeklärten „Common Sense“ (ebd., XIX, XXII) teilen können. Die biologisch nötige Reduktion dieser Qualitäten auf experimentell Beobachtbares und Berechenbares stelle zwar einen verwertbaren Gewinn dar, könne aber nicht die Erfahrung von Qualitäten in der Lebensführung freier Personen ersetzen. Insofern lege die Philosophie des lebendigen Daseins jene „Voraussetzungen“ und „Vorbedingungen“ frei, die Biologie und ihre Anwendung in der Lebenswelt ermöglichen, wie umgekehrt die Biologie von diesen lebensweltlichen Kontexten durch methodische und theoretische Reduktion zugunsten der experimentellen und berechenbaren Reproduzierbarkeit der Phänomene befreie (Plessner 1975, 72, 88, 114, 301). Daher bräuchten beide Seiten, Biologie und Philosophie, ihre Kooperation (ebd., 65f., 116), in der keine Seite die andere ersetzen kann.

\subsection{Leibliches Ausdrucksverhalten. Verweise auf Uexkülls Biologie in Die Deutung des mimischen Ausdrucks (1925)}

In Die Deutung des mimischen Ausdrucks. Ein Beitrag zur Lehre vom Bewusstsein des anderen Ichs entwirft Plessner mit Buytendijk einen neuen philosophischen Zugang zu einer vergleichenden Verhaltensforschung, die möglich werde, wenn man „Verhalten“ nicht auf „behavior“ (Plessner 1982c, 79) reduziere, sondern auf alles öffentlich beobachtbare „Benehmen“ erweitere: „Zur Konstitution des Tierkörpers gehört, schon rein phänomenal, die Schicht des Verhaltens. Umwelt und Leib sind hier gegensinnig aufeinander bezogen, indem das Tier in seiner Ortsbewegung sich als Lebenszentrum gegen eine Außensphäre absetzt und mit ihr in Wechselbeziehung bleibt“ (ebd., 114). Im Unterschied zur tierlichen Verhaltenseinheit zwischen Organismus und ihm korrespondierender Umwelt seien aber die Verhaltensweisen von Menschen als Personen durch zusätzliche und Distanz gebietende Differenzen charakterisiert, weshalb sie in einer „Welt“ lebten: Die Dinge (aktionsrelative Konstanten in der Wahrnehmung) aus der Umwelt nehmen in der Welt den Charakter von Gegenständen an, d. h. eines begrifflich bestimmten Zusammenhangs zwischen dem sinnlich nicht gegebenen „Substanzkern“ und seinem sinnlich gegebenen „Eigenschaftsmantel“ (ebd., 115). Aber nicht nur auf Seiten des Objekts gebe es eine strukturelle Verdoppelung in die Differenz zwischen einer sinnlichen „Gegebenheit plus einen nicht aktuellen Ungegebenheitsrest“, sondern auch auf der Subjekt-Seite spalte sich das Bewusstsein in ein „mit sich in innerer Zwiesprache lebendes Ich“ (ebd., 114f.) auf. So stehe der Mensch als Person „in einer Umwelt, die nach der Ichseite wie nach der Esseite aufgebrochen in die Unendlichkeit einer Welt 
weist“ (ebd., 114: Hervorhebung im Original). Dieser Stellung eines jeweils in sich verdoppelten Subjekts und Objekts entspreche die sprachliche Verbindung von Subjekt und Objekt im Verhalten: Im Verhalten von Personen werden sprachlicher Ausdruck und Sprachhandlung miteinander verbunden (ebd., 92f.).

Gleichwohl bedeute diese sprachliche Spezifik des Verhaltens von Personen in einer Welt nicht, dass sie aufhörten, sich als Lebewesen zu verhalten. So können die sprachlichen Zeichen als Stellvertreter für Ausdrücke, Gegenstände, Personen und deren Handlungen nicht die Ausdrücke und Handlungen von Organismen und Dingen ersetzen. Man dürfe nur nicht diese sprachliche Spezifik von personalem Verhalten in der Welt auf die Tier-Umwelt-Relationen übertragen. Uexkülls Kritik am „Anthropomorphismus“ (ebd., 80) sei berechtigt, ebenso seine Betonung der Eigengesetzlichkeit der Biologie gegenüber der Physik (ebd. 83). Aber seine Kritik erfolge vom Standpunkt des „Physiologen“ (ebd., 80), der die „moderne Tierpsychologie“ („die Untersuchungen Thorndikes, Morgans, Volkelts und Köhlers“ ebd., 116) aus den Möglichkeiten der vergleichenden Verhaltensforschung ausklammert. Richtig an der physiologischen Kritik des Anthropomorphismus sei, „dass ein belebter Körper zwar Wirkungen empfängt und ausübt, aber niemals sieht oder greift. Das kann allein ein Leib, d. h. ein lebendiger Körper, zu dessen Einheitsgestalt die Richtung auf die Gegenwelt einer gestalteten Umgebung mitgehört“ (ebd., 80: Hervorhebungen im Original). Für Plessner, so seine Grundbestimmung, die dann Maurice Merleau-Ponty wortwörtlich übernimmt, stellt der Leib die „Umweltintentionalität“ (ebd., 79) des Organismus in seinem Verhalten zur Umwelt dar (Merleau-Ponty 1966, 272).

Plessner entwirft philosophisch unter dem Titel des Leibes eine Möglichkeit, die Verhaltenseinheit von Subjekt und Objekt neu zu thematisieren. Diese Thematisierung liege vor der Aufspaltung der Untersuchung in entweder die Physiologie oder die Psychologie des Verhaltens. Er will gerade auf einen künftigen Zusammenhang zwischen Tier-Physiologie und Tier-Psychologie hinaus. Dafür knüpft er unter anderem an Uexkülls eigene Einsicht in den ganzheitlichen Charakter des Verhaltens von Organismen an. Uexküll spreche treffend von einer „Bewegungsmelodie“ (Plessner 1982c, 84) im Verhalten, um den ganzheitlichen Charakter der Tonfolge in den Folgeschritten einer Bewegungsrichtung gleichsam hörbar zu machen. Dieses musikalische Beispiel passe zur leiblichen Verhaltensschicht, weil es nicht zwischen der Realität der Bewegung in einem Bild und der sinnhaften Richtung dieser Bewegung als reiner Form trennt, sondern die „ursprüngliche Identität von Anschaulichkeit und Verständlichkeit auf Grund des Formcharakters der Bewegungsgestalten“ verdeutliche 
(ebd., 83). Während Personen in ihrer Sprache, Mathematik und Logik klar unterscheiden können zwischen einem Anschaulichen, von dem man ein sinnliches Bild habe, und einem intentional Verständlichen, das man als solches nicht sinnlich wahrnehmen kann, zeichne sich das leibliche Verhalten gerade dadurch aus, dass es gegen die Trennung zwischen einem sinnlichen Bild für Reales und dessen geistigem Sinn im Ganzen „indifferent“ (ebd., 84) sei. Indem die reale Bewegung sinnlich bildhaft anhebe, entfalte sie auch schon ihre intentional sinnhafte Richtung wie eben in einer Melodie. „Entscheidend für die Möglichkeit des Armhebens oder des Gehens ist das Ergreifen und Eingreifen in die leibhaft gegebene Körpergestalt, die sowohl der physiologischen als auch der psychologischen Erklärungsrichtung vorausliegt“ (ebd.).

Man versteht die folgenden Indifferenzen, die laut Plessner der leiblichen Verhaltensschicht spezifisch sind, wenn man sich die Problemlage vor Augen hält, die durch den Nachweis der praktischen Intelligenz von Schimpansen durch die Tierpsychologie entstanden war: Wolfgang Köhler hatte gezeigt, dass Schimpansen im Rahmen ihrer erlernbaren Trieberfüllungen Intelligenz, d. h. die plötzliche Einsicht in eine neue Problemlösung, entwickeln können. Dabei handelte es sich um eine in das praktische Verhalten eingelagerte Intentionalität, die die Schimpansen nicht sprachlich (oder anders symbolisch) von ihrem Verhalten abheben konnten. Um diese Art und Weise von Umweltintentionalität im tierlichen Verhalten zugänglich werden zu lassen, dürfe man nicht, so Plessner, die sprachliche Reflexion über reine, d.h. nicht sinnlich erfahrbare Intentionalität im Gegensatz zum sinnlich erfahrbaren Verhalten auf die höher entwickelten Tiere anthropozentrisch übertragen. Man müsse die Umweltintentionalität im tierlichen Verhalten freischaufeln von den Dualismen, d.h. den exklusiven Entweder-Oder-Alternativen, die erst in der Moderne durch geistige Selbstreflexion entstanden und institutionalisiert worden sind.

Daher postuliert Plessner den Zugang zur Umweltintentionalität des Leibes, indem er diesen Untersuchungsgegenstand gegen die folgenden exklusiven Differenzen „neutralisiert“ (im Anschluss an und in Radikalisierung gegen Max Scheler: ebd., 127f.): Die leibliche Verhaltensschicht sei nicht entweder sinnlich oder geistig, nicht entweder physisch oder psychisch, nicht entweder objektiv oder subjektiv, sondern ursprünglich beides, nämlich die lebendige Verbindung zwischen Sinn und Geist, Physis und Psyche, Objekt und Subjekt im sichVerhalten. Die leibliche Verbindung von Organismus und seiner Umwelt sei mehr

[...] als ein Inbegriff von Gestalten, obwohl sie sich immer in Gestalten darstellt, und deren Überschuss über das rein Gestaltmäßige als objektive Sinnhaftigkeit erscheint, so dass Anschauen und Verstehen an ihr eine gemeinsame Sphäre für die Betrachtung besitzen. 
Was sich in der Schicht des Verhaltens abspielt, ist gegen die sphärentheoretischen Begriffspaare Sinnlichkeit und Geistigkeit, Physis und Psyche, Objektivität und Subjektivität indifferent. Nur das, was ein Seiendes ist, gehört irgendeiner dieser Sphären an. In der Schicht des Verhaltens jedoch erfassen wir den Inbegriff der Konstitutionsformen und weisen alles Seienden: das Leben. (Ebd., 89: Hervorhebung im Original, siehe auch 125)

Diese Neutralisierung des Untersuchungsverfahrens für leibliches Verhalten klammert die Verselbständigung der Produkte moderner Reflexion ein. Sie führt aus der modernen Anthropozentrik heraus, in der sich das eigene Selbst als Selbstbewusstseins im eigenen Körperleib für primär hält (ebd., 116f.), da es sich von allem anderen durch dualistische Hebel abgespalten hat. Dagegen seien zwei Einsichten zu stellen: „1. Die Gewissheit der Du-Form und der DuRealität ist gleichursprünglich mit, weil gegensinnig zu der Gewissheit der IchForm und der Ich-Realität; 2. Die Leibhaftigkeit ist nicht nur die Seins-, weil Auffassungsweise des eigenen Körpers durch den sich erlebenden Menschen, sondern der Seins- und Auffassungsmodus der Körper in der Schicht des Verhaltens oder das psychophysisch indifferente Schema, wonach Körperbilder von Subjekten füreinander und miteinander erst möglich sind“ (ebd., 125: Hervorhebung im Original). Diese beiden Einsichten würden möglich, wenn man das Ich aus seiner falschen Verortung im eigenen Körper befreit und in die leibliche Zwischenschicht zwischen Subjekt und Objekt, zwischen Subjekt und Subjekt verlegt. Das Verstehen der Körperbilder müsse immer durch die jeweilige Situation, in der sie auftreten, ergänzt werden. Die Einklammerung der modernen Privilegierung des eigenen Selbst erfordere vor allem eine alternative Konzeption für die „Mitwelt“ von Personen, deren „Überindividualität“ und „Intersubjektivität“ (ebd., 128), die Plessner in dieser Schrift noch nicht ausführen konnte.

Diese philosophische Öffnung der Verhaltensforschung soll im leiblichen Ausdruck „Seinswerte“ und im leiblichen Handeln „Funktionswerte“ (ebd., 91) ermitteln, die strukturell, funktional und situativ variabel sind. Vom Standpunkt dieser Perspektive künftiger Verhaltensforschung schätzt Plessner Uexkülls mechanische Biologie als zu begrenzt ein: In der modernen Biologie habe Uexküll den Versuch unternommen, ,alle psychischen Aktivitäten als Beziehungen von Innenwelt und Umwelt aufzufassen, wobei innerhalb einer konstanten Form sowohl der Umwelt wie auch des in sie eingepassten Organismus allein der Inhalt dieser Beziehungen bei den verschiedenen Tieren und beim Menschen wechseln soll.“ (ebd., 74) Aber eine „derartige Nivellierung nach dem Schema Merkwelt-Innenwelt-Wirkungswelt“ werde „niemals dem Bedeutungswechsel der Außenwelt, d. h. der Variabilität der inneren und äußeren Form und damit der Reizwerte gerecht.“ (Ebd.: Hervorhebung im Original) In der Tat hatte Uexküll seinen in der Form konstanten, allein im Inhalt variab- 
len Funktionskreis anhand von wirbellosen Tieren mit gelegentlichem Vergleich niedrig entwickelter Wirbeltiere ausgeführt, ohne diesen Funktionskreis für höher entwickelte Wirbeltiere und den Menschen überzeugend durchführen zu können (siehe Uexküll 1973, 158 u. 198; Uexküll 2014, 63-65).

\subsection{Plessners Auseinandersetzung mit Uexkülls Biologie in Die Stufen des Organischen und der Mensch (1928)}

Das naturphilosophische Hauptwerk von Plessner enthält seine ausführlichste Auseinandersetzung mit Uexkülls Biologie. Erneut stimmt Plessner Uexkülls Kritik an „Anthropomorphismen“ im Allgemeinen zu, um die Biologie im modernen Sinne als eine streng „experimentelle Untersuchung“ $\mathrm{zu}$ ermöglichen. Dies bedeutete jedoch unter der dualistischen Voraussetzung, dass uns fremde Körper durch die allein sinnliche Wahrnehmung von außen zugänglich werden, während der direkte Zugang zum Psychischen eine „introspektive Selbstbeobachtung“ erfordere, eine eingeschränkte Lösungsrichtung:

Infolgedessen forderten Forscher wie Beer, Bethe und v. Uexküll eine objektive, alle Anlehnungen an psychologische Begriffe meidende Terminologie der Lebensforschung, Uexküll besonders statt Tierpsychologie Biologie als eine die objektiv kontrollierbaren Zuordnungen von Reiz und Reaktion im Bauplan des jeweiligen Tieres feststellenden Wissenschaft. Nicht die ewig verborgen bleibende Innenwelt der Tiere mit ihrem uns unzugänglichen Empfinden und Befinden, sondern die Umwelt, d. h. die jeweilig verschiedene Gestalt der Einheit derjenigen Momente, die für sie wirksam werden und auf die sie wirken können, sei das wissenschaftliche Programm des ,Tierpsychologen'. Keine Kryptopsychologie, sondern Phänologie des lebendigen Verhaltens: Erklärung des uns sichtbaren Gebahrens der Tiere aus sinnlich wahrnehmbaren Faktoren. (Plessner 1975, 63)

Plessner selbst geht es indessen um eine „Revision des cartesianischen Alternativprinzips im Interesse der Wissenschaft vom Leben“ (ebd., 63). Daher stellt er die Frage,

[...] ob man es beim Uexküllprogramm mit einem Maximal- oder Minimalprogramm zu tun hat. Wäre es ein Maximalprogramm, so müssten sich alle Fragen, welche die sogenannte Tierpsychologie aufgeworfen hat, als Probleme der Reiz- und Bewegungsphysiologie erledigen. Tatsächlich nimmt aber Uexkülls Biologie (Lebensplanforschung) das Programm gar nicht in diesem Sinne. Sie ist auf die Erforschung der für den Organisationstyp des betreffenden Tieres je charakteristischen Reize und Reaktionen beschränkt, nicht weil sie die Zielsetzung einer vergleichenden Psychologie (im Sinne des dogmatischen Mechanismus) ablehnt, sondern sie mit Rücksicht auf die mangelnden Mittel für undurchführbar hält. Sie stellt durchaus nicht in Abrede, dass sich dem Gedanken des ,Lebensplans' noch eine andere Seite abgewinnen lässt. Nur bestreitet sie ihre empirische Erforschbarkeit, oh- 
ne damit die Möglichkeit einer solchen (nicht empirischen) Untersuchung von vornherein abzuschneiden. (Ebd., 64: Hervorhebungen im Original)

Dies ergebe sich daraus, dass zur „Idee des Plans“ eine Ganzheit gehöre, „die mehr als die Summe der ihn realisierenden Faktoren“ darstelle, ohne dass es sich bei dieser Planmäßigkeit um einen „Zweck- und Zielcharakter“ handeln muss:

Lebensplan als Einheit der Reize, die erkennbar vom Organismus beantwortet werden, und eben seiner Antwortreaktionen kann darum nicht mit der Summe derartiger wahrnehmbarer Vorgänge identisch sein. Diese an den Vorgängen sichtbar werdende, selbst unsichtbare Einheit der Sphäre, die den vorgegebenen Rahmen für Reize und Reaktionen bedeutet, gehört damit weder dem Körper des Organismus noch der ihn umgebenden Welt allein an. Sinnes- und Bewegungsorgane können, wenn es solche ,Pläne‘ gibt, nicht ,vor` der Welt der Dinge sein, für die sie da sind - und umgekehrt. (Ebd.: Hervorhebungen im Original)

Mit dieser, nicht sinnlich wahrnehmbaren Ganzheit im jeweiligen Lebensplan wird für Plessner der Weg frei zu jenen Kategorien einer philosophischen Biologie, die die impliziten Voraussetzungen der empirischen Biologie explizieren. Solche „Kategorien“ ermöglichen empirische „Begriffe“, in denen die vorausgesetzte Ganzheit des Lebendigen analysiert werden kann, ohne in die Resultate der Analyse zu zerfallen (ebd., 116).

Jede empirisch feststellbare Eingepasstheit des Organismus in die Umwelt, Angepasstheit der Umwelt an den Organismus, weist eben auf übergreifende, Lebenssubjekt und Welt gleichmäßig beherrschende Gesetzmäßigkeiten hin. Dass keines der beiden Glieder dieses Gegenseitigkeitsverhältnisses den Vorrang vor dem anderen hat, lässt sich jedoch selbst nicht mehr empirisch begreifen. (Ebd., 64f.)

Mit „Gesetzmäßigkeit“ ist keine im Sinne einer Kausalerklärung gemeint, sondern eine solche des Verstehens anschaulicher Tatbestände im Ganzen:

Kategorien sind demnach Formen, die weder dem Subjekt noch dem Objekt allein angehören und sie vermöge ihrer Neutralität zusammenkommen lassen. Sind Bedingungen der Möglichkeit des Übereinkommens und der Eintracht zweier wesensverschiedener und voneinander unabhängiger Größen, so dass diese weder durch eine unüberbrückbare Kluft getrennt sind noch direkt aufeinander Einfluss haben. (Ebd. 65)

Breche man hingegen mit dem cartesianischen Alternativprinzip, brauche man nicht mehr das Bewusstsein vom Standpunkt des Selbstbewusstseins zu analysieren und im Resultat dieser Analyse das Bewusstsein „als eine unsichtbare Kammer oder Sphäre, als ein unräumliches Pendent zum räumlichen Gehirn“ aufzufassen, „das mit diesem immerhin die Binnenexistenz im Kopf hinter den 
Sinnesorganen gemeinsam habe“ (ebd.). Die Tierintelligenz und auch das vorreflexive Bewusstsein von Menschen zeige, dass Bewusstsein „nicht Selbstbewusstsein“ (ebd.) zu sein brauche. „In Wirklichkeit ist die Sache gerade umgekehrt“ zu der Verdinglichung des Bewusstseins in einer Kammer, die im Kopf liegen soll: „nicht ist das Bewusstsein in uns, sondern wir sind ,im“ Bewusstsein, d. h. wir verhalten uns als eigenbewegliche Leiber zur Umgebung“ (ebd.). Mit der Verlegung des vorreflexiven Bewusstseins in das leibliche Verhalten befreie man auch die Tierpsychologie von der Fehlorientierung, durch eine Introspektion in die Psyche der Tiere gelangen zu müssen.

Dementsprechend kümmert sich die junge Wissenschaft der Tierpsychologie nicht um Erlebnisse, sondern sucht sich als eine Lehre vom tierischen Verhalten, seinen Formen und Faktoren, zu entwickeln. Zwischen der Scylla anthropomorphisierender Seelenschilderung von Klugheit, Treue und Liebe und der Charybdis des Uexküllprogramms, das alle Bewusstseinserforschung in Verruf erklärt, führt eine schmale Straße. Sie vermeidet auf diese Weise sowohl den aus Objektivitätsdrang überängstlichen und ganz in der physiologischen Reiz-Reaktionsschematik befangenen ,Behaviorismus', [...] als auch die unkritische Allbeseelungs- und Vermenschlichungsromantik der Laien. (Ebd., 68)

Zusammenfassend würdigt und begrenzt Plessner daher Uexkülls Forschungsprogramm wie folgt:

Uexküll war der erste, der das Verhältnis Organismus-Umwelt als Arbeitsgebiet einer zur Vernunft gebrachten Tierpsychologie (Biologie, Lebensplanforschung) proklamierte. Die junge Wissenschaft ist jedoch insofern über ihn hinausgegangen, als sie dieses Verhältnis (im Gegensatz zum ,Kantianer‘ Uexküll) in seiner Lebendigkeit und Verständlichkeit zu erfassen strebt und nicht mehr die physiologischen Bedingungen, an die sein Zustandekommen geknüpft ist, mit dem Gesamthabitus des Benehmens der Tiere identifiziert. W. Köhler, dem wir die hervorragenden Untersuchungen über Intelligenz der Anthropoiden verdanken, David Katz und F. J. J. Buytendijk treiben wohl am entschiedensten Tierpsychologie in dieser Richtung: mit vollem Bewusstsein der rein bildmäßigen Natur der Basis dieser Wissenschaft, von dem reinen Phänomencharakter des ,Verhaltens‘. (Ebd., 69)

Da das lebendige Benehmen mindestens Gestaltcharakter habe und „nur im Habitusbild gegeben“ sei, müsse es vor allem im Falle „niederer Tiere“ nicht unmittelbar verständlich sein. Die experimentelle Methodik schließe eine „physiologische Beobachtungskontrolle“ ein, „aber gerichtet auf die Bildeinheiten lebendigen Verhaltens“ (ebd.).

Nach dieser Einschätzung von Uexkülls Beitrag zur Forschungslage in der Biologie entfaltet Plessner seine eigene Naturphilosophie. Da er in ihrer Durchführung stellenweise auf Uexküll zurückkommt, muss zunächst der naturphilosophische Kontext dieser Stellen verdeutlicht werden, um die Bemerkungen zu Uexküll verstehen zu können. 
Plessners kategoriale Unterscheidung lebender Körper von anorganischen Körpern erfolgt ganz unabhängig von Uexküll. Ihr gemäß leben Körper insofern, als sie in ihrem Verhalten ihre eigene Grenze vollziehen (ebd., 103f.). Sie gehen aus sich heraus in ein Medium oder Umfeld hinaus und von dort und dann zurück in sich. Dieser Vollzug des Grenzüberganges werde in der Anschauung durch Sinnrichtungen fassbar, die zwischen dem Raum und der Zeit im physikalischen Sinne und der Raum- und Zeitlosigkeit rein geistiger Gehalte liegen. In dieser Raumhaftigkeit entfalte sich der Sinn einer Bewegung, in dieser Zeithaftigkeit erfülle sich der Sinn einer Bewegung (des Beutefangs, der Flucht etc.). Da aber diese Anschauung bloßer Schein sein könnte, wird sie nicht nur phänomenologisch untersucht, sondern auch hermeneutisch in der Ontologie des lebendig Seienden verstanden: Unter welchen ontologischen und ontischen Bedingungen kann diese Anschauung im sich-Positionieren des Körpers wirklich sein (ebd., 122, 129)? Um diese Frage beantworten zu können, deduziert Plessner seine philosophischen Kategorien so, dass sie den qualitativen Gehalt der Anschauungen von Lebewesen explizieren und gleichzeitig empirische Begriffe der Biologie ermöglichen. Dazu gehören zunächst die wesentlichen Struktur- und Funktionsbedingungen der dynamischen Realisierung der eigenen Grenze, d. h. der „Prozesscharakter und die Typenhaftigkeit“ (ebd., 132) des lebendigen Seins, der „Entwicklungscharakter des lebendigen Prozesses“ (ebd., 138). Sodann folgen die Bedingungen der „statischen Realisierung“ der eigenen Grenze im „Systemcharakter des lebendigen Einzeldinges“ (ebd. 154f.), seiner „Selbstregulierbarkeit“ (ebd., 160) und seiner „Organisiertheit“ (ebd., 165).

Unter den statischen Realisierungsbedingungen müsse man, so Plessner, zwischen zwei Weisen unterscheiden, in denen das Ganze in den Teilen eines lebendigen Einzeldinges vertreten werde: „Das Ganze des lebendigen Körpers ist unmittelbar selbst in seinen Teilen potentiell vorhanden. Diese seine Form der Vertretung heißt das harmonisch äquipotentielle System“ (ebd., 168), das Plessner unter Verweis auf die von Hans Driesch behandelten Phänomene der Selbstregulation darstellt. Dabei hält er wie schon früher (und so auch Uexküll) Drieschs Annahme, bei der Entelechie handele es sich um einen Naturfaktor, für vollkommen unnötig: „An Stelle der Entelechie als Naturfaktor tritt Entelechie als Seinsmodus entsprechend jener Grenzbedingung, die sich selbst noch verstehen lässt, obzwar für sie keine physikalische Charakterisierung (,Erklärung`) gegeben werden kann“ (ebd., 146). Das Ganze sei aber nicht nur unmittelbar als Potenz (Vermögen) in den Teilen vorhanden, sondern auch vermittelt als Aktualität: „Diese seine Form der Vertretung liegt vor in der harmonischen Divergenz spezialisierter Organe“ (ebd., 168). Diese Form erläutert Plessner unter Verweis auf Uexküll, der unter Organisation „den Zusammenschluss verschiedenartiger 
Elemente nach einheitlichem Plan zu gemeinsamer Wirkung“ verstanden habe (ebd., 170; vgl. Uexküll 2014, 184-187).

Plessner hält diese systemtheoretische Konzeptualisierung des Problems, wie es aktual zur Vergegenwärtigung des Ganzen in den Organen kommen kann, nämlich durch Vermittlung im Bauplan der Verhaltensfunktionen, für angemessen, um die statischen Realisierungsbedingungen der eigenen Grenze aufzuklären:

Das Ganze des Organismus ist nicht nur logisch, sondern ontologisch jener doppelten Abhebung von ihm als physischem Körper fähig, ja, er konstituiert sich geradezu in und mit dieser Abhebung, die in den Worten ,zu' und ,nach' gefasst wird. Erst als Einheit von Zweck und Mittel ist der lebendige Körper Ganzheit oder autonomes System. (Plessner 1975, 171)

Umso wichtiger wird dann die Frage, wie die Organisation „nach“ einheitlichem Plane und „zu“ gemeinsamer Wirkung in den Sinnrichtungen der Raumhaftigkeit und der Zeithaftigkeit verstanden werden kann. Im Unterschied zur Reversibilität der Richtungen im physikalischen Raum und der physikalischen Zeit gebe es für die Leibesrichtungen der Lebewesen in ihrer Raumhaftigkeit und Zeithaftigkeit eine Irreversibilität. Für einen bestimmten Leib sind sein Vorne und Hinten, sein Links und Rechts, sein Unter und Über ihm, seine Altersstufen im Ganzen nicht umkehrbar, sondern unumkehrbar. Dies bedeute in der Richtung seiner Zeithaftigkeit eine Fundierung aus der Zukunft in die Gegenwart und Vergangenheit, also eine Umkehrung der kausalen Sukzessionsfolge aus der Vergangenheit über die Gegenwart in die Zukunft (vgl. ebd., 176). In seinen Potenzen sei das Sein des lebendigen Körpers ihm selber so vorweg, dass es situationsspezifisch aktualisiert werden könne, indem die Erfüllung des Bezuges zum Modus der Gegenwart durch die Erfüllung des Bezuges zum Modus der Zukunft bedingt werde (ebd., 177).

In Rückbindung von der Zukunft her steht der lebendige Körper, ihm selber vorweg, d. h. Zweck, seinem dauernden Übergehen vom Noch nicht ins Nicht mehr entgegen oder beharrt. Das abstrakte Jetzt zwischen Zukunft und Vergangenheit eignet sich nicht mehr zum Schema seiner Existenz, sondern nur die konkrete Gegenwart, deren Differential der Augenblick ist, Einheit von Zukunft und Vergangenheit. Darum hat weiterhin der lebendige Körper, in Rückbindung das ihm selber Nachseiende, eine Vergangenheit. (Ebd., 180: Hervorhebungen im Original)

In der Auseinandersetzung mit der Theorie der natürlichen Evolution geht es Plessner um die Aufdeckung der kategorialen Voraussetzungen für die biologischen Begriffe Anpassung und Selektion: Um hier und jetzt in einer bestimmten Situation von einer aktualen Anpassung sprechen zu können, müsse man struk- 
turell und funktional eine „Angepasstheit“ in dem Verhältnis zwischen dem Organismus und seiner Umwelt voraussetzen (ebd., 200-205). Auch das aktuale Selegieren hier und jetzt erfolge nicht ex nihilo, sondern unter der Voraussetzung einer Selektivität in struktureller und funktionaler Hinsicht (ebd., 211-216). Zudem können sowohl Anpassung/Angepasstheit als auch Selektion/Selektivität von beiden Seiten aus, sowohl vom Organismus als auch seiner Umwelt her, verstanden werden. Erst dank dieses Kategoriennetzes und der in ihm möglichen Erfüllungsrichtungen fallen einem die Einschränkungen im Züchtungsmodell Darwins auf, in dem sich der Organismus an die Umwelt anpassen soll (nicht umgekehrt) und der Organismus von der Umwelt selegiert werden soll (nicht umgekehrt) (ebd., 206). Plessner denkt den Rahmen des philosophischen Kategoriennetzes als ein Spektrum an strukturell-funktionalen Spielpotentialen, des Ein-Spielens und der Eingespieltheit in beide Richtungen, sowohl des Organismus auf die Umwelt als auch umgekehrt der Umwelt auf den Organismus.

Im Rahmen dieses Kategoriennetzes kommt Plessner auch auf Uexkülls Biologie zu sprechen. So schreibt er, ganz im Sinne Uexkülls, von der „Eingepasstheit des Organismus in die Umwelt“ und der „Angepasstheit der Umwelt an den Organismus“ (ebd., 64; vgl. Uexküll 1973, 319-321; Uexküll 2015, 169). Uexküll habe zwar in seinen Lebensplanformen die „primären Harmonien“ an Eingepasstheit sehen gelehrt, aber nicht ohne „damit dem entgegengesetzten Extrem einer absoluten Angepasstheit des jeweiligen Lebenssystems, einer biologischen Monadologie gewissermaßen [...] zuzuneigen“ (Plessner 1975, 207). Die struktur-funktionale Angepasstheit in der Form des Verhaltens ersetze nicht die aktuale Anpassung im Verhalten hier und jetzt angesichts eines bestimmten Inhalts. Der Organismus bleibe bei aller „Geborgenheit gefährdet. Das Positionsfeld oder das Milieu ist wesensmäßig Schauplatz von Kämpfen und Sphäre des Schutzes“ (ebd.). „Vorgreifend in der Form, suchend im konkreten lebendigen Akt nach Kontakt mit dem Medium, angepasst-anpassend spielt sich das Leben des Organismus im Verhältnis zum Umfeld ab. Dabei lässt sich die Form vom konkreten Einzelinhalt nicht lösen“ (ebd.). Auch könne man „die Fülle der Baupläne nicht aus der Bauplangesetzlichkeit überhaupt“ entwickeln, diese Fülle setze vielmehr eine „spielerische Willkür“ im Leben voraus: „Das belebte physische Ding trägt an sich die ordnungsschaffenden Bedingungen, die mit dem begonnenen Spiel selbst hemmend und fördernd ins Spiel treten“ (ebd., 170). Im Gegensatz zu Plessner endet Uexkülls theoretische Biologie in einer „überwältigend großen Planmäßigkeit“, der „alle Pläne“ angehören sollen (Uexküll 1973, 342; Uexküll 2014, 237). 
Die geschlossene Organisationsform des Tieres zeichne sich, gegenüber der offenen Organisationsform von Pflanzen, dadurch aus, dass nervöse Zentren den Gegensatz zwischen sensorischen und motorischen Organen zur Einheit vermitteln. Die Ermöglichungsbedingung für das Realsein dieser geschlossenen Form habe Uexküll in seinem „Funktionskreis“ verständlich gemacht, sowohl morphologisch und damit ontogenetisch als auch physiologisch (Plessner 1975, 230), weshalb Plessner auch Uexkülls Schema des Funktionskreises modifiziert übernimmt (ebd., 248). Aber Uexkülls Unterscheidung zwischen Merken und Wirken setze kategorial einen Leib voraus, der sich etwas merken und etwas bewirken kann (ebd., 231). Uexküll bleibe bei der physiologischen Verdoppelung des Körpers stehen, die durch die Repräsentation der Organe im Zentralorgan entstehe, weil er die Beschreibung der psychischen Innenwelt der Tiere für empirisch unmöglich halte. Demgegenüber gelte es aber zu erkennen, dass „der positionale Gegenwert jener physischen Trennung in eine das Zentrum mit enthaltende und eine vom Zentrum gebundene Körperzone“ (ebd., 237) im Doppelaspekt des tierlichen Verhaltens von außen (nicht in der für innen gehaltenen Psyche) liegt. Nicht als Gesamtkörper (einschließlich des Zentralorgans), sondern nur als Leib (als vom Zentralorgan abhängige Körperzone) könne das Tier zu dem „Subjekt des Habens“ oder einem „Selbst“ werden, d. h. handeln (ebd., 237f.), d. h. den Zusammenhang zwischen Merken und Wirken zustande bringen. Plessner vermeidet daher in seiner Modifikation von Uexkülls Schema den Ausdruck „Innenwelt“ ganz, weil es auch ihm nicht um die Erforschung einer inneren Psyche geht. Zudem ersetzt er Uexkülls Ausdrücke „Merkwelt“ und „Wirkungswelt“ durch „Merksphäre“ und „Wirkungssphäre“, weil er in dem Ausdruck „Sphäre“ Uexkülls Gedanken von der Einheit zwischen Subjekt und Objekt gewahrt sieht, ohne Uexkülls Fehlidentifikation von „Welt“ mit „Umwelt“ zuzustimmen (ebd., 248).

Was Uexküll das „Gegengefüge“ in dem „Objekt“ der Umwelt (Uexküll 2014, 45) nennt, durch das die Wirkungsträger und die Merkmalsträger überhaupt zu ein und demselben Objekt gehören können, dient Plessner als Ausgangspunkt für eine weitere Problematisierung von Uexkülls Annahmen. Um wie Uexküll sagen zu können, dass der Wurm nur Wurmdingen in seiner Umwelt begegnen könne, bedürfe es eines biologischen Beobachters, der die Einheit des Objekts als desselben Trägers von verschiedenen Merkmalen und Wirkungen feststellen kann. Uexküll könne die Einheitlichkeit dieses Gegenstandes nicht aus der „Gegenwelt“ des Tieres heraus entwickeln, in der laut ihm selbst gerade nicht die Umwelt gespiegelt werde, sondern der Spiegel der Gegenwelt selber den Ausschlag gebe. Es handele sich also nur um eine „Projektion“ der tierlichen „Gegenwelt“ auf das „Gefüge“ von Objekten in der Umwelt (ebd., 
169). Plessner lehnt diesen „zoologischen Idealismus“ von Uexküll klar ab, weil er an die Stelle eines weltschöpferischen Bewusstseins (Henri Bergson) nur „eine weltschöpferische Organisation“ setze (Plessner 1975, 259). Demgegenüber vertritt Plessner die Relation der „Koexistenz“ von Organismus und Umwelt (ebd., 258): an „dieser Relation besitzt kein Glied über das andere Übergewicht“ (ebd., 259). Plessner hält dem Konstruktivismus, hier im Falle Uexkülls in der Gestalt des Bauplans, nicht den Standpunkt der „Abbildung“ von Objekten entgegen, sondern den Standpunkt der strukturalen und funktionalen „Entsprechung“ zwischen dem Bewusstsein der Tiere und ihrer Umwelt (ebd., 260). Plessners „Korrelationsstufentheorie von Lebensform und Lebenssphäre“ (ebd., IV) verbindet durchgängig eine Art von Konstruktivismus mit der entsprechenden Art von ontologischem Realismus in der Art und Weise des sichAusdrückens: „Der Mensch kann nur erfinden, insoweit er entdeckt“ (ebd., 321).

Für Plessner unterschätzt Uexküll die psychischen Leistungen des Bewusstseins höher entwickelter Tiere, weil er sich an die damalige „Unkenntnis“ in der Neurophysiologie halte (siehe Uexküll 2014, 308). Uexküll berücksichtigt zwar in seiner theoretischen Biologie die für Lernprozesse wichtige Art der „kontrollierten Handlung“: „Es stellt sich dabei die Notwendigkeit heraus, die Tätigkeit der eigenen Effektoren durch die eigenen Rezeptoren zu kontrollieren. Die kontrollierte Handlung ist eine Erfahrungshandlung, die nicht zum Reflex wird“ (Uexküll 1973, 307). Aber während Uexküll diese Handlungsart an Menschen erklärt, ist sie für Plessner längst an Menschenaffen sicher nachgewiesen. Er nennt den zentralnervösen Anschluss der Aktionen eines Tieres an sein eigenes Sensorium, so dass es seine Handlung kontrollieren kann, den „Ringschluss des sensomotorischen Funktionsspiels“, dem im Verhalten das „Auftreten von Dingen im Merkfeld entspricht“ (Plessner 1975, 255). Dieser Ringschluss im Zentralorgan des Gehirnes stelle die physiologische Bedingung dafür dar, dass das Tier „seine Bewegungen im Umfeld“ merkt, mithin sich als seinen Leib in der von ihm selbst eingenommenen Zone merkt, wodurch das Umfeld mit eigener Grenze von seinem Leib abrückt und eine „Struktur“ bekommt, nämlich die Struktur von Dingen, die Merkmale und Wirkungen tragen. Das Umfeld des Tieres werde so zu einem „Signalfeld und Aktionsfeld in Einem“ (ebd., 252), was Uexkülls Schema vom Funktionskreis eigentlich voraussetze, ohne es selber für höhere Tiere erklären zu können.

Uexküll werte dieses Potential, dass „der Kreis der sensomotorischen Funktionen“ im „Zentralorgan noch einmal geschlossen“ (ebd.) wird, noch unter dem Primat aus, dass so Sensorik und Motorik besser, weil im Erlernen plastischer miteinander verbunden werden können. Für Plessner ist aber das Gehirn schon neurophysiologisch gesehen nicht nur eine bessere Möglichkeit, Sensorik 
und Motorik hier und jetzt miteinander zu verbinden, was er bereits den niedriger, weil dezentral organisierten Tieren zuspricht. Für Plessner ist das Gehirn hingegen vor allem ein Potential dafür, diese Verbindung auch zu unterbrechen, was sich gerade bei höher entwickelten Säugern zeige. Denke man die Zentralisierung (statt Dezentralisierung des Nervensystems) in der Schließung der Organisationsform gegenüber der Umwelt konsequent zu Ende, dann verselbständige sich das Gehirn (Neokortex) gegenüber seinen sensorischen und motorischen Funktionen: Das Gehirn sei neurophysiologisch gesehen das Korrelat gerade für „die Unterbrechung, Hemmung, Pause (zwischen Reiz und Reaktion), welche positional das Sein eines Selbst in Mittelstellung, d. h. sein , gegen etwas im Umfeld' Sein oder seine Anschauung von Etwas ist“ (ebd., 260f.: Hervorhebungen im Original). Eben diese Unterbrechung oder Pause im aktualen Verhaltensvollzug ermögliche dem Tier auch, seine gegenwärtigen Erlebnisse zu sieben, sich etwas vorzustellen, was noch nicht da ist (ebd., 268), und ein Gedächtnis für die ihm relevanten Fragmente seiner Vergangenheit auszubilden (ebd., 284).

Obwohl Uexküll das Bewusstsein der Tiere von Dingen, ihrer Griffigkeit, Konstanz und Haltbarkeit, unterschätze, weil er eine Hirnphysiologie des Korrelates dafür nicht sehe, habe er doch zurecht auf die Bedeutung von „Gleichgewichtsorganen“ aufmerksam gemacht, die es dem Organismus ermöglichen, ein eigenes raumzeitliches Koordinatensystem gegenüber seiner Umwelt auszubilden (ebd., 257). Im Ganzen gesehen ist Plessner davon überzeugt, dass eine Hirnphysiologie der Pausen in der Verbindung von Sensorik und Motorik zur „objektiven Disziplinierung der Interpretationen“ in der Tierpsychologie beitragen wird:

Je differenzierter Rezeptoren und Gehirn, desto vielfältiger die anklingenden Erregungen, desto mannigfaltiger die Pausen und damit die Struktur des Positionsfeldes. Nervöse Erregungen des sensorischen (und motorischen) Apparates schaffen dem Lebewesen nur die jeweiligen Gelegenheiten, jene Mittelstellung einzunehmen, als welche und in welcher sein bewusstes Leben sich abspielt. (Ebd., 261)

Zum Abschluss entwickelt Plessner seine Unterscheidung zwischen der Umwelt von Tieren in dem, was er die zentrische Positionalität nennt, und der Welt von Menschen oder anderen personalen Lebewesen in dem, was er kategorial als die exzentrische Positionalität bezeichnet, ohne noch einmal speziell auf Uexküll Bezug zu nehmen, wohl aber, um die Voraussetzung zu explizieren, die die Biologen machen müssen, ohne sie als Biologen einholen zu können. Dieses Problems war sich Uexküll in dem Schlusskapitel seines Buchs Umwelt und Innenwelt der Tiere bewusst, das den Titel der Beobachter trägt; womit der Bio- 
loge gemeint ist, der außerhalb der Umwelt der Tiere steht (Uexküll 2014, 233). Einerseits erscheint die Weltauffassung des Biologen als eine Voraussetzung dafür, dass er die Umwelt der Tiere als einen Ausschnitt daraus erkennt: „Jede Umwelt eines Tiers bildet einen sowohl räumlich wie zeitlich, wie inhaltlich abgegrenzten Teil aus der Erscheinungswelt des Beobachters“ (ebd., 236). Andererseits soll aber die Welt des Menschen, darunter des Biologen, auch nur die Umwelt eines Lebewesens sein: „Die Erscheinungswelt eines jeden Menschen gleicht ebenfalls einem festen Gehäuse“, nämlich wie schon die Umwelt des Tieres ein „undurchdringliches Gehäuse“ für es sei, „,das ihn von seiner Geburt bis zum Tode dauernd umschließt“ (ebd., 237). „Das gleiche gilt für die Erscheinungswelt des Beobachters, auch diese schließt ihn, da sie seine Umwelt darstellt, völlig vom Universum ab“ (ebd.).

Es bleibt bei Uexküll offen, wie man dieses sich-Gleichen von Umwelt und Welt verstehen soll: Handelt es sich nur um ein lebensphilosophisch bekennendes Gleichnis zwischen Menschen und Tieren, die vor der Natur alle gleich sind, das aber offenbar doch nur Menschen, nicht Tiere ziehen können; oder soll die Biologie für das Vergleichen von Umwelt und Welt wirklich einen Maßstab entwickeln, der sich empirisch und rechnerisch realisieren lässt, wenngleich Uexküll selber dies nicht zu leisten vermag? Für Plessner stellt ein derart biologischer Vergleichsmaßstab klar einen kategorialen „Kardinalfehler“ dar, an dem gemessen der Mensch höchstens als „gesundes“ oder als „krankes Tier“, als „Raubtier“ oder als „Haustier“ erscheine, ohne die Spezifik seiner personalen Lebenssphäre erfassen zu können (Plessner 1975, 315, 318).

Plessner nennt eine Lebenssphäre genau dann eine exzentrische Positionalität, wenn in ihr die Exzentrierung von der Konzentrik des Leibes wirklich möglich wird (ebd., 307). Die Konzentrik des Leibes ist auf die „Konvergenz“ mit etwas in seinem Umfeld ausgerichtet (ebd., 291f.). Die Mitte der zentrischen Positionalität liegt da und dort, wo und wann sich das leibliche Verhalten in der Begegnung mit etwas in der Umwelt erfüllt, z. B. wenn der Hund sein Bällchen zum Apportieren gerade schnappt. Exzentrierung dieser Mitte erfordert, eine Distanz von ihr zu gewinnen, aus der man diese Mitte als Gegenstand erkennen kann. Plessner nennt den Standpunkt, der außerhalb der leiblichen Einheit zwischen dem Organismus und der Umwelt liegt, den der „Person“ (ebd., 293). Sie kann ihren Organismus als Körper verwenden, d. h. instrumentieren und als Medium gebrauchen, um die leibliche Einheit ihres Organismus mit etwas in der Umwelt herbeizuführen. Dafür muss die Person aber in einer Mitwelt mit anderen Personen stehen können, die ihren Geist teilen. Der Geist stellt das Nichts von Bestimmungen in der physikalischen Raumzeit dar und das Nirgendwo und Nirgendwann von Bestimmungen in den leiblichen Sinnrichtungen. Dadurch 
wird der Kontrast möglich zwischen dem, was sinnlich gegeben ist, und dem, was es begrifflich bedeutet, ein Kontrast, der in Symbolen kulturell in Verbindungen ausgelegt wird. Vom Standpunkt der geistig geteilten Mitwelt können die Personen interpretieren, wie sie sich in ihrer Außenwelt, d. h. in der physikalisch reversiblen Raumzeit und der leiblich irreversiblen Raum- und Zeithaftigeit, und in ihrer Innenwelt, d. h. in ihrer Gegenstandsstellung und in ihrer Selbststellung, wirklich verhalten sollten, müssten und könnten. Da Personen als Glieder einer geistig geteilten Mitwelt nicht aufhören, Lebewesen zu sein, d. h. Körper zu haben und Leib zu sein, müssen sie die Welt, die sie sich geistig eröffnet haben, auf künstliche und geschichtlich vermittelte Weise einrichten. Für die personale Lebenssphäre ist wesentlich ihr geschichtlicher Aufgabencharakter, den Plessner in drei anthropologischen Grundgesetzen erläutert: dem der „natürlichen Künstlichkeit“ (ebd., 309), dem der „vermittelten Unmittelbarkeit“ (ebd., 321) und dem des „utopischen Standorts“ (ebd., 341, siehe ausführlich Krüger 2017c und im vorliegenden Band 3. Kapitel).

\subsection{Die geschichtliche Verschränkung von soziokultureller Umwelt und Welt im personalen Leben und die öffnung biotisch geschlossener Umwelten (1938-1961)}

Plessner wiederholt seinen Grundstandpunkt in den verschiedensten Diskussionen von 1938 bis 1961: „Jede biologische Umweltinterpretation muss in letzter Instanz auf einem außerbiologischen Weltbegriff ruhen“ (Plessner 1983j, 59). Die biologische Erkenntnis von tierlichem Verhalten in bestimmten Umwelten erfolgt nicht innerhalb dieses tierlichen Verhaltens, sondern setzt einen Abstand von diesem Verhalten voraus, um es als Gegenstand thematisieren zu können. Diese „Konzession“ an den menschlichen Weltbegriff müsse auch Uexküll erlauben, wenn er als Biologe schildere, „wie er durch Fliegen-, Spinnen-, Hundeaugen und mit Fliegen-, Spinnen- und Hundeinteressen die Welt sieht“: „Nur weil sie Welt, nicht bloß Umwelt ist, gibt die menschliche Welt den konstanten Hintergrund ab“ (ebd.). Dem stehen aber die Aussagen innerhalb der Uexküllschen Biologie entgegen:

Mit diesem Gedanken eines Pluralismus der Umwelten, der einen Pluralismus der biologischen Wertmaßstäbe einführt, den Gedanken des Fortschritts, der steigenden Anpassung an die eine einzige Umwelt unmöglich macht, die Intelligenz relativiert, wird natürlich auch die Sonderstellung des Menschen in der Natur hinfällig, wenn die Welt, in der der Mensch lebt, nichts anderes als die Umwelt des menschlichen Bauplans ist. (Ebd.) 
Die Sonderstellung des Menschen in der Natur beruht für Plessner darauf, dass die Gattung der Menschheit am logos, d. h. an einer geistigen Mitwelt teilhat, die es gestatte, die personalen Verhaltensweisen „vernünftig“ (ebd., 55) und im Zeichen selbstzweckhafter wie zweckfreier Liebesbeziehungen auszubilden (Plessner 2001d, 167). Man dürfe dieses geistige Selbstverständnis des Menschen in einer Welt nicht mit der „instinktgebundenen Intelligenz“ von Tieren verwechseln, d. h. mit einem Verhalten, das zwar „für Korrekturen durch Erfahrung offen ist“, sich aber „auf eine jeweils spezifische Umwelt“ erstreckt, „in deren Konstellationen sie Einblick gewährt“ (Plessner 1983j, 56). Im Unterschied zur instinktgebundenen Intelligenz für biotisch spezifische Umwelten eröffne der geistig-allgemeine Abstand von Körpern und Leibern eine Welt von sprachlich-symbolisch darstellbaren Potentialen, in deren Rahmen und aus deren Hintergrund es gelte, „eine künstliche Umwelt zu schaffen“ (ebd., 63). Diese geschichtliche Aufgabe setze ein „Nichtfestsitzen des Menschen“, der an keine biotisch spezielle Umwelt mehr vorangepasst sei, voraus und erfordere die „Fähigkeit der Ausschaltung und der Unterbrechung des biologischen Zusammenhangs“ in dem „Umkreis von Trieben und Instinkten“ (ebd., 64). Dieser Sonderstellung des Menschen in der Natur kommen biologische Einsichten über den Menschen entgegen: So habe L. Bolk die bleibende Ähnlichkeit von Menschen mit dem fötalen Stadium anderer Anthropoiden und das lange Kindheits- und Jugendstadium von Menschen hervorgehoben. P. Alsberg habe die Ergänzung und Ersetzung natürlicher durch künstliche Organe, insbesondere durch Sprache, beim Menschen herausgearbeitet (ebd., 60, 62f.). „Fundmental verstanden ist der Mensch der Emigrant der Natur, die keine Heimat von Natur hat, sondern nur insoweit, als er sie sich erobert und mit allen seinen geistigen Kräften des Gedankens und des Herzens an ihr festhält“ (ebd., 64).

Plessner versucht, aus dem falschen Gegensatz zwischen nur geschlossener Umwelt und allein offener Welt herauszuführen: „Die heute übliche Zuordnung geschlossener Umweltbindung zum Tier und der Weltoffenheit zum Menschen macht sich die Sache zu einfach“ (Plessner 1983e, 182). Die Eröffnung von Welt lasse sich nicht ohne die Einrichtung einer künstlichen Umwelt stabilisieren, die ihrerseits von den irdischen Lebensbedingungen abhängig bleibt. Personale Lebewesen würden im Unterschied zu Engeln, die wohl in reiner Weltoffenheit existieren mögen (ebd., 187), von Körpern und Leibern abhängig bleiben, indem sie in ihnen etwas und jemanden, also geistige Gehalte, darstellen. Unter dem Zwang im personalen Leben, „sich der offenen Wirklichkeit zu stellen und ihrer Unvorhersehbarkeit Herr zu werden, ergibt sich überall eine künstliche Horizontverengung, die wie eine Umwelt das Ganze menschlichen Lebens einschließt, aber gerade nicht abschließt. Die künstliche Horizontverengung ist 
vielmehr die Art und Weise vermittelnder Unmittelbarkeit, welche das ganze menschliche Verhalten charakterisiert" (ebd., 189). Statt einfach von Weltoffenheit zu reden, spricht Plessner, wie schon in seinen „Stufen“ (Plessner 1975, 293), vom „fragmentarischen Charakter menschlicher Weltoffenheit“ (Plessner 1983e, 188). Aber nicht nur die Kategorie der Welt ist in sich durch die Kategorie der soziokulturellen Umwelt zu vermitteln, auch die Umwelt im biologischen Sinne muss nicht immer als nur geschlossen veranschlagt werden. Nur insofern der physiologische Bauplan aus Uexkülls geschlossenem Funktionskreis bestehe und das Verhalten des Tieres entsprechend bestimme, sei diese Umwelt auch geschlossen. Allein dann gelte für die entsprechenden Tiere, dass ihnen ihre Umwelt nicht transponierbar sei, weil ihr Bauplan sie zur Selektion aus dem Umfeld nötigt, da er ihre Aktionsrelativität gegen alles dafür Irrelevante isoliert (ebd., 182f.). Aber schon die Säuger und insbesondere die Anthropoiden stellten den Gegenbeweis einer schrittweisen Öffnung der biotischen Umwelt für Intelligenz, Werkzeugherstellung und soziale Mitverhältnisse dar, umso mehr die anzunehmende Evolutionsgeschichte des Menschen.

Was die zuletzt genannte biologische Möglichkeit angeht, wie man in der Anpassung an die Umwelt auch eine „Unabhängigkeit“ von ihr gewinnen kann (ebd., 166), hebt Plessner Folgendes hervor: Unter den Säugern liege auf der „Linie aufsteigender Zerebralisierung“ und intelligenter Verarbeitung der Erfahrung auch das Wachstum der „Jugendphase“ als einer „Zeit des Lernens und Spielens“ vor. Buytendijk habe gezeigt, wie sich in dieser frühen ontogenetischen Lage ein Triebüberschuss bilde, der nach Abfuhr drängt, aber eine unfertige Motorik und Sexualität vorfinde: „Daraus resultiert das Spiel, ein zweckfreies Verhalten zwischen Bindung und Lösung, das eine von Angst und Gier unbelastete, in sich selbst erfüllte Beziehung stiftet“ (ebd., 167). Beim Menschen steigere sich der Freiheitsgewinn gegenüber der biotischen Umwelt durch seine Schaffung einer soziokulturellen Umwelt als Welthorizont. Das Ende seiner Embryonalentwicklung finde schon außerhalb des Uterus in der soziokulturellen Umwelt (A. Portmann) statt, in der bereits im letzten Drittel seines ersten Lebensjahres Erwerbsmotorik (im Unterschied zur Erbmotorik), Spracherwerb und transponierbare Handlungsintentionalität gleichzeitig zu greifen beginnen (ebd., 166, 182). Mit der Einlagerung der Sprache in den aufrechten Gang und die Auge-Hand-Koordination integriere ein nur mehr „virtuelles Organ“ (ebd., 177) den menschlichen Verhaltensaufbau, dessen geistige Leistung keiner biologischen Funktion mehr unterliege (ebd. 181). „Imitation und Vergegenständlichung, von denen Spracherwerb und Sprachgebrauch leben, haben die gleiche menschliche Wurzel: Sinn für die Reziprozität der Perspektiven im Verhältnis von meinem leibhaften Dasein zum Dasein des anderen“ (ebd. 179). 
Gleichwohl bedürfe diese Eröffnung von Welt durch Symbolformen einer stabilen Reproduzierbarkeit der Verhaltensweisen in der künstlichen Formung von Körpern und Leibern. Körper und Leiber werden für Personen zu Medien, in denen sie Sachverhalte und interpersonale Beziehungen darstellen. Solche Medien der Darstellung und Aufführung nennt Plessner die „Rollen“ von Personen, in denen und mit denen man Gemeinschaft und Gesellschaft spielt (ebd., 193, 205, 209). Seine geschichtliche Schauspieltheorie der Personenrollen habe ich andernorts ausführlich dargestellt, insbesondere die Verdoppelung der Person in eine private und öffentliche (Krüger 1999, im vorliegenden Band Zweiter Teil). Hinsichtlich des Verhältnisses von soziokultureller Umwelt und Welt bleibt hier Plessners Kritik an Erich Rothackers Kulturanthropologie hervorzuheben, in der die soziokulturelle Umwelt wieder an eine biotisch „echte“ Umwelt angenähert wird:

\begin{abstract}
Aber die Geschlossenheit nach außen und Offenheit nach innen, die Vertrautheit und Selbstverständlichkeit eines geistigen Lebensraumes, in dem wir Menschen in jeweils anderen Überlieferungen groß werden und gewöhungsmäßig gefangenbleiben können, wenn wir uns ihrem Bannkreis nicht zu entziehen vermögen oder ihm entrissen werden, geben kein Recht, ihn als eine echte Umwelt zu bezeichnen, weder im Singular noch im Plural. So wie ein geistiges Gerüst aus Sprache, Werten, Gütern und Sitte in all seiner Geschlossenheit und Unübertragbarkeit zugleich auch nach außen offen bleibt - zwischen Sprachen gibt es die Möglichkeit der Übersetzung - und Brücken zu anderen geistigen Gerüsten der Vorzeit und Mitwelt bildet, Durchblicke in fremdes Geistesleben gewährt, hebt es sich deutlich von den auch umwelthaften Bindungen rein vitalen und emotionalen Charakters ab, in denen wir Menschen mit unserer Tiefenperson, vorbewusst, affektiv, instinktiv leben. (Plessner 1983e, 187)
\end{abstract}

Die „ganze Umweltbildung beim Menschen“ habe ein „erworbenes und bewahrtes Wesen“, das „nicht mit der Natur seines Leibes einfach gegeben, sondern weil kraft ihrer offen gelassen - gemacht und nur im übertragenen Sinne natürlich gewachsen“ (ebd., 184) sei.

Am Ende seines Versuchs, durch neue Differenzierungen aus der falschen Gegenüberstellung von geschlossenen Umwelten der Tiere und der Weltoffenheit des Menschen heraus zu gelangen, verdeutlicht Plessner seinen eigenen Standpunkt (auch im Gegensatz zu anderen philosophischen Anthropologen wie M. Scheler und A. Gehlen): „Mit der Möglichkeit, das Umweltgebundenheit und Weltoffenheit kollidieren und nur im Verhältnis einer nicht zum Ausgleich $\mathrm{zu}$ bringenden gegenseitigen Verschränkung gelten, rechnet weder die klassische noch die pragmatistische Anthropologie“ (ebd., 182). Die Überraschungen der Geschichte bestehen aus solchen Kollisionen, in denen der Ausgleich nicht mehr gelingt, selbst wenn man die Not, Umwelt und Welt gegenseitig verschränken zu müssen, glaubt eingesehen zu haben (zu Plessners Geschichtsphi- 
losophie im vorliegenden Band Dritter Teil): Die ökologische Problemlage ist heute ein Beispiel dafür.

1965, im Nachtrag zur zweiten Auflage seiner Stufen, stellt Plessner fest, dass Uexkülls „doppelte Frontstellung gegen die mit anthropomorphen Analogien arbeitende Tierpsychologie und die an Kettenreflexmodellen orientiert gewesene amerikanische Behavioristik“ jede Aktualität für die moderne Ethologie verloren habe (Plessner 1975, 354). Das Differenzierungspotential seiner Biologie für die Phänomene zwischen der Tierpsyche und den Kettenreflexen war zu gering. Demgegenüber haben Plessners Differenzierungen zwischen der Intelligenz, dem Bewusstsein und dem Geist in verschiedenen Lebenssphären, zwischen dem Mitmachen, dem Nachmachen und dem Nachahmen von Sachund Person-Zusammenhängen in der Reziprozität von Rollen erneut an Aktualität für die vergleichende Hirn- und Verhaltensforschung gewonnen (Krüger 2010 u. 2014b, siehe im vorliegenden Band 8. bis 10. Kap.). 